\title{
Stability and Efficacy Enhancement of Vitamin C Powder via Developing a Serum - Two Phase System, A New Inception in Vitamin C Delivery
}

\author{
Vishal Saxena ${ }^{1 *}$, Krishana Yadav $^{2}$ \\ ${ }^{I}$ Department of Pharmacy, B. N. University, Udaipur, India \\ ${ }^{2}$ Department of Pharmacy, The Maharaja Sayajirao University of Baroda, Vadodara, India
}

\begin{abstract}
Vitamin C (Vit C) is a potent antioxidant with several applications in the cosmetic and pharmaceutical fields. However, the biggest challenge in the utilization of Vit $\mathrm{C}$ is to maintain its stability and improve its delivery to the active site. Several strategies have been developed such as: controlling the oxygen levels during formulation and storage, low $\mathrm{pH}$, reduction of water content in the formulation and the addition of preservative agents. In this article, we highlighted the multiple applications of Vit $\mathbf{C}$ and the development of novel serum formulation to enhance its delivery and stability during the application.
\end{abstract}

Keywords: Vitamin C (Vit C), serum, pigmentation stability.

\section{Introduction}

Vitamin $\mathrm{C}$ has been proved as an important and potent antioxidant and anti-aging ingredient in topical formulations [1]. It is found in the living organisms in its reduced form ascorbic acid or in its oxidized form called dehydroascorbic acid [2]. Vit $\mathrm{C}$ is able to reduce unstable species of oxygen, nitrogen and sulfur radicals in addition to regenerating other antioxidants in the body, such as alpha-tocopherol (Vitamin E). Besides, studies with human plasma have shown that Vit $\mathrm{C}$ is effective in preventing lipid peroxidation induced by peroxide radicals [3]. When applied topically, Vit $\mathrm{C}$ can neutralize reactive oxygen species (ROS) triggered by exposure to solar radiation and environmental factors such as smoke and pollution. Studies also indicate that Vit $\mathrm{C}$ is effective in the treatment of hyperpigmentation, melasmas and sunspots [4].

The major challenge for the utilization of Vit $\mathrm{C}$ is to maintain its chemical stability. Vit $\mathrm{C}$ is easily oxidized in aqueous media, in alkaline $\mathrm{pH}$ (higher stability is obtained at $\mathrm{pH} \leq 4$ ) and in the presence of light, oxygen and metal ions [5]. This process is usually accompanied by a color change in the formulations, which become gradually more yellowish. Several strategies have been developed to limit these processes, among them: controlling the presence of oxygen during formulation and storage, low $\mathrm{pH}$ and reduction of water content through the use of anhydrous/nonaqueous formulations [6]. The addition of preservatives such as antioxidants and anti-chelating agents also prevents the degradation of the Vit C.
In the current research work a 2 Phase system was developed wherein an anhydrous Ascorbic Acid powder form was used and a water based activating serum was used separately. Separating Vitamin C from water phase helps to improve the stability of Vit C. The current approach is providing a unique mode of delivery for such type of materials which are not much stable in aqueous media and frequently get degraded in formulation upon keeping for long time. Thus, we developed a separate water based activating serum without $\mathrm{Vit} \mathrm{C}$, which can be incorporated by mixing Vit $\mathrm{C}$ powder at the time of application to avoid the degradation of Vit $\mathrm{C}$.

\section{Method}

The activating serum was developed using xantan gum as thickener, and rest of the preservatives were added as per the requirement. 100\% Ascorbic Acid was used in the powder system. The powder and activating serum is developed to be mixed in 1:4 ( $0.2 \mathrm{~g}$ of Vitamin $\mathrm{C}$ powder and $0.8 \mathrm{ml}$ of activating serum). The combination delivers $25 \%$ active Ascorbic Acid. The present research was conducted on 12 volunteers having mild to severe pigmentation. The 12 volunteers comprised of 5 males and 7 females and were divided into 3 groups on the basis of their skin variability. Skin lightening effect was observed by visual inspection. The treatment was for an average of 6 weeks and comprised of series of applications every week with proposed formulation (developed serum + Vit C). The 6-weeks monitoring of each volunteer showed a visible skin lightening effect without any side effects. The effect starts after 3 weeks in all the 12 volunteers. The subjects were required to use 2 applications of the system every day for 6 weeks.

\section{Discussion and Conclusion}

During the applications, the volunteers were asked to follow all possible precautions that can hamper the treatment results. Volunteers were informed about possible side effects of sunexposure and were advised to use sun protection creams every day during the treatment period and also informed to stay inhouse for at least 24 hours after the treatment day. The proposed 
formulation (developed serum + Vit C) used in the study has shown significantly visible results on skin complexion. Typical skin cell turn-over rate is 4-5 weeks but topical application of proposed formulation (developed serum $+\mathrm{Vit} \mathrm{C}$ ) has shown to decrease this cycle, making Vit $\mathrm{C}$ the most preferred skin lightener. As shown in figure 1, volunteers have seen a visible improvement after 3 weeks of treatment. The proposed formulation (developed serum + Vit C) has shown considerable effects on skin lightening without any side effects.

Figure $1,2 \& 3$ shows the effect of skin lightening after 6 weeks.

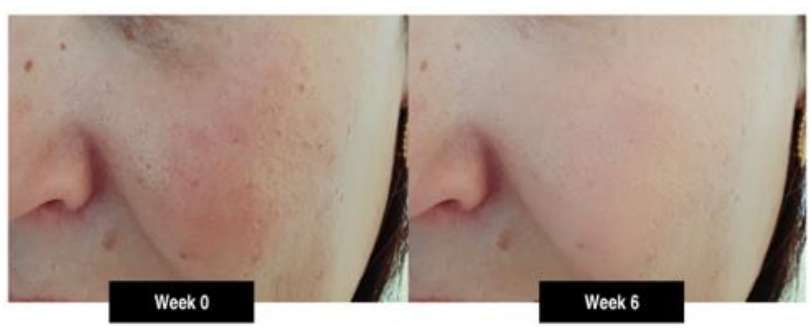

Fig. 1. Before and after effects of 2 phase system after 6 weeks in Volunteer 1

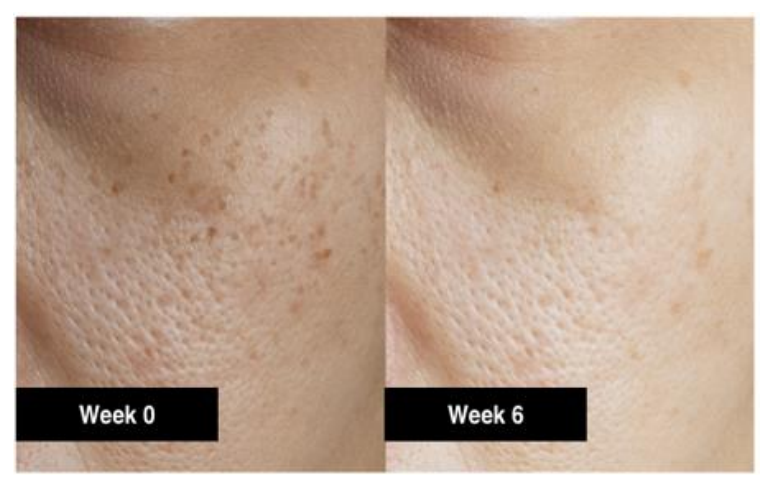

Fig. 2. Before and after effects of 2 phase system after 6 weeks in Volunteer 2

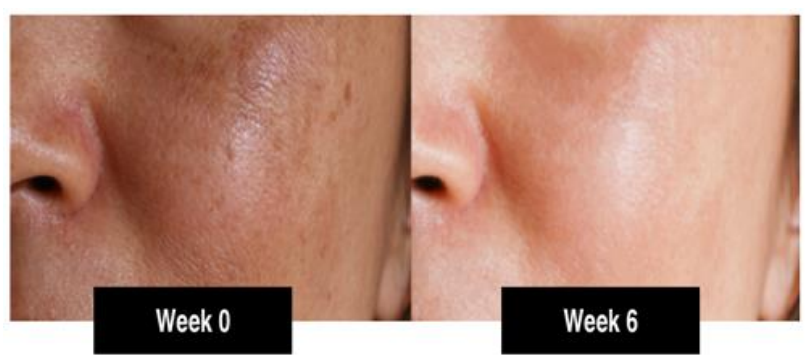

Fig. 3. Before and after effects of 2 phase system after 6 weeks in Volunteer 3

\section{References}

[1] Ak M. "A comprehensive review of acne vulgaris". J Clin Pharm. 1:1745, 2019.

[2] Tang SC, Yang JH. "Dual effects of alpha-hydroxy acids on the skin". Molecules. 23(4):863, April 2018.

[3] Alba MN, Gerenutti M, Yoshida VM, Grotto D. "Clinical comparison of salicylic acid peel and LED-Laser phototherapy for the treatment of Acne vulgaris in teenagers". Journal of Cosmetic and Laser Therapy. 2;19(1):49-53, January 2017.

[4] Green BA, Wildnauer RH, Edison BL. "Polyhydroxy acids (PHAs) provide conditioning effects to skin without increasing sensitivity to UV light". Amer Acad of Derm Poster Exhibit. February 2002.

[5] Wang C. M, Huang C.L, Sindy Hu C. T, Chan H. L, "The effect of glycolic acid on the treatment of acne in Asian skin" Dermatologic surgery. 23(1):23-9. Jan.

[6] Pullar, J. M, Carr, A. C, Vissers, M. C. M, "The roles of vitamin C in skin health" Nutrients. 9: 866, 2017. 